Revue française de la traduction

\title{
La circulation des sciences humaines et sociales en traduction : enjeux et obstacles à l'heure de la globalisation
}

Gisèle Sapiro

\section{OpenEdition}

Journals

Édition électronique

URL : http://journals.openedition.org/traduire/465

DOI : $10.4000 /$ traduire.465

ISSN : 2272-9992

Éditeur

Société française des traducteurs

Édition imprimée

Date de publication : 15 décembre 2012

Pagination : 5-15

ISSN : 0395-773X

\section{Référence électronique}

Gisèle Sapiro, «La circulation des sciences humaines et sociales en traduction : enjeux et obstacles à I'heure de la globalisation », Traduire [En ligne], 227 | 2012, mis en ligne le 01 décembre 2014, consulté le 02 mai 2019. URL : http://journals.openedition.org/traduire/465 ; DOI : 10.4000/traduire.465 


\section{La circulation des sciences humaines et sociales en traduction : enjeux et obstacles à l'heure de la globalisation}

\section{Gisèle Sapiro}

À la différence de la littérature, fortement liée à la langue et à la nation, dont elle a contribué à construire l'identité culturelle, la science n'a pas attendu la mondialisation pour revendiquer son caractère transnational et tend à adopter un langage commun plus ou moins formalisé, l'anglais servant, comme autrefois le latin, de langue de communication privilégiée. Les sciences humaines et sociales occupent une position intermédiaire entre ces deux pôles, la proximité à I'un ou à l'autre variant selon les disciplines. Certaines disciplines ou sous-disciplines (comme la littérature comparée ou le droit international) se caractérisent, en effet, par une circulation internationale élevée(1). Néanmoins, l'ancrage de la plupart d'entre elles dans des traditions nationales(2), soit sur le plan théorique, soit du point de vue des objets, demeure indéniable : c'est le cas des plus "littéraires ", mais aussi des sciences nées de la demande étatique, comme la science politique ou, dans certains pays, la sociologie.

Quels sont les effets de la mondialisation et de la construction européenne sur cet (inter) nationalisme propre aux sciences humaines ? C'est la question à laquelle on tentera d'apporter ici quelques éléments de réponse en prenant pour point d'observation les conditions de circulation des livres de sciences humaines et sociales en traduction. Après un rappel des transformations du marché de la traduction depuis les années 1980, on se concentrera sur les enjeux de la traduction d'ouvrages de science humaine et sociale.

\section{La globalisation du marché de la traduction}

La plus ancienne des industries culturelles n'a pas attendu la " globalisation " pour franchir les frontières. Antérieur à la constitution des États-nations, le marché du livre se structurait, à

(1) Yves Gingras, "Les formes spécifiques de l'internationalité du champ scientifique ", Actes de la recherche en sciences sociales, $n^{\circ} 164,2002$, p. 31-45.

(2) Johan Heilbron, "Repenser la question des traditions nationales en sciences sociales ", in Gisèle Sapiro (dir.), L'Espace intellectuel en Europe. Des États-nations à la mondialisation, Paris, La Découverte, 2009, p. 310-318. 
I'origine, autour des aires linguistiques avant d'être divisé par des frontières nationales qui, se superposant aux précédentes, ont fixé un cadre juridique et douanier. À partir du milieu du XIXe siècle, la traduction devient le principal mode de circulation, le processus de construction culturelle des identités nationales(3) impliquant un phénomène d'intemationalisation. Le marché de la traduction est régulé, à partir de 1886, par la Convention de Berne sur le droit d'auteur, à laquelle nombre de pays se rallient au tournant du xxe siècle. Dans l'entre-deux-guerres, un ensemble d'acteurs - agents, éditeurs, traducteurs, critiques, représentants étatiques - et d'instances - agences de presse, agences littéraires, collections - commencent à se spécialiser dans la médiation interculturelle. Interrompus par la Deuxième Guerre mondiale, les échanges s'intensifient après la fin du conflit, s'ouvrant, à l'initiative de l'UNESCO, aux pays non occidentaux (Extrême-Orient, Amérique latine).

Le mot d'ordre de la globalisation visant à l'ouverture des frontières en vue du libre-échange des biens et des services a accéléré ces échanges tout en relativisant le rôle des États au profit des logiques propres au marché. On constate ainsi une intensification de la circulation des livres en traduction : entre 1980 et 2000, l'ensemble des traductions dans toutes les langues a augmenté de $50 \%$, passant de 50000 à près de 75000 livres traduits (réimpressions incluses), selon l'Index Translationum de l'UNESCO. Mais dans quelle mesure cette intensification s'est-elle accompagnée d'une diversification?

Le nombre de traductions faites à partir d'une langue constitue un indicateur de sa centralité sur le marché de la traduction. Plus une langue est centrale, plus on traduit d'ouvrages de cette langue ; plus elle est périphérique, moins de livres en sont traduits(4). Au début des années 1990, les traductions de l'anglais concentraient environ $44 \%$ des traductions dans le monde ; l'allemand, le français et le russe représentaient entre 10 et $12,5 \%$ du marché mondial des traductions. Huit langues, dont l'espagnol et l'italien, occupaient une position semi-périphérique, avec une part variant entre $1 \%$ et $3 \%$. Toutes les autres langues se situaient dans une position périphérique, avec un pourcentage inférieur à $1 \%$. En une décennie, la domination de l'anglais s'est accrue, passant à $59 \%$ des titres traduits, selon l'Index Translationum, tandis que le taux du russe a chuté après 1989 à moins de $3 \%$ (l'allemand et le français se maintiennent autour de 9-10\%). L'espagnol est une des rares langues à avoir connu une hausse de $1,7 \%$ à $2,6 \%$. Le japonais approche désormais les $1 \%$ grâce aux mangas. Les langues asiatiques ont, de manière générale, renforcé leur position, le chinois en particulier, tout en demeurant en deçà du seuil de $1 \%$.

Ces évolutions montrent que les flux de traduction ne sont pas le simple reflet de la taille des marchés, mais dépendent d'autres facteurs, politiques et culturels, qui contribuent à des formes

(3) Anne-Marie Thiesse, La Création des identités nationales. Europe XVIe siècle-xxe siècle, Paris, Le Seuil, 1999.

(4) Johan Heilbron, "Towards a Sociology of Translation. Book Translations as a Cultural World System", European Journal of Social Theory, 2/4, 1999, p. 429-444. 
d'hégémonie : conquête de marché, influence politique (perte d'influence du russe après 1989), hégémonie culturelle, consécration, les fonctions de la traduction peuvent être multiples selon les types d'intérêts qui sont investis dans cette activité.

Sous ce rapport, le rôle des politiques publiques est central. La chute du russe après 1989 est due en grande partie à l'interruption des politiques de soutien à la traduction (qui étaient importantes non seulement à destination des pays communistes mais aussi des pays arabes, et au sein de l'URSS, entre les différentes langues). II est frappant que la part de $10 \%$ perdue par le russe corresponde à peu près à la hausse des traductions de l'anglais. Après 1989, la zone d'Europe de l'Est a été fortement investie par les éditeurs anglo-américains comme un nouveau débouché pour leurs produits. Les traductions de littérature dite " commerciale " de l'anglais ont afflué dans cette zone(5).

Du point de vue des types de livres produits, le marché du livre se structure en effet, selon l'opposition entre d'un côté un circuit de grande diffusion, à rotation rapide, où la production obéit à une logique industrielle et à la quête de rentabilité, de l'autre un circuit de diffusion restreinte, à rotation lente, où prédominent le mode de production artisanal et les critères intellectuels(6). D'un côté, les best-sellers et autres genres commerciaux, (roman rose, guides touristiques, livres pratiques, etc.), qui se vendent à des dizaines voire des centaines de milliers d'exemplaires. De l'autre, les ouvrages scientifiques et les œuvres littéraires, qui ne dépassent qu'exceptionnellement les 10000 exemplaires lorsqu'il s'agit de nouveautés, mais qui peuvent atteindre un public beaucoup plus large une fois qu'lls sont devenus des classiques. Certains marchés, comme ceux des États-Unis et du Royaume-Uni, sont segmentés selon cette opposition, l'édition commerciale se démarquant de l'édition à but non lucratif (non profit), qui inclut les presses universitaires et les éditions subventionnées par des fondations, comme The New Press, la maison fondée par André Schiffrin.

Le circuit de diffusion restreinte se caractérise par son faible rendement sur le court terme. Les logiques qui président à son fonctionnement sont d'ordre intellectuel et symbolique plus qu'économique, même si, à terme, le capital symbolique peut se convertir en capital économique lorsque l'ouvrage devient un classique ; mais pour cela il faut la médiation du champ intellectuel et/ou du champ académique. Ce modèle s'applique aussi bien aux ouvrages traduits qu'aux livres publiés dans leur langue d'origine et peut être étendu au marché mondial du livre(7). Alors

(5) Elżbieta Skibińska, "La place des traductions sur le marché éditorial polonais ", in Gisèle Sapiro (dir.), Les Contradictions de la globalisation éditoriale, Paris, Nouveau Monde, 2009, p. 335-368.

(6) Pierre Bourdieu, "La production de la croyance : contribution à une économie des biens symboliques ", Actes de la recherche en sciences sociales, $n^{\circ}$ 13, 1977, p. 3-43; " Une révolution conservatrice dans l'édition ", Actes de la recherche en sciences sociales, $n^{\circ} 126 / 127,1999$, p. 3-28.

(7) Gisèle Sapiro, "Translation and the field of publishing. A commentary on Pierre Bourdieu's 'A conservative revolution in publishing' from a translation perspective", Translation Studies, vol. 1, n 2, 2008, p. 154-167. 
que le circuit de grande production est fortement rationalisé et professionnalisé, les agents littéraires y jouant un rôle central, l'économie symbolique du secteur de diffusion restreinte se caractérise par un fort investissement des acteurs (auteurs, éditeurs, traducteurs), qui y participent souvent sur le mode vocationnel et désintéressé, et la quête de profits symboliques ou militants plus qu'économiques. Du fait de son faible rendement, le fonctionnement de ce secteur repose souvent sur une politique publique et des aides de l'État ou d'organisations.

Or si la période de la " globalisation " a été marquée par l'unification progressive d'un marché mondial de la traduction et par le développement de l'édition commerciale dans des pays où elle était encore confinée à un appareil idéologique d'État, comme on l'a vu dans le cas de la Russie, elle a également vu s'accroître les contraintes économiques qui pèsent sur le commerce du livre, sous l'effet des mouvements de concentration (fusions/acquisitions), de la formation de grands groupes internationaux et du phénomène concomitant de financiarisation. On constate ainsi que, pour la littérature, l'augmentation des traductions s'est faite surtout au profit des genres les plus commerciaux (best-sellers, thrillers, etc.) et de la littérature pour la jeunesse, devenue un secteur prolifique depuis le début des années 1990.

L'opposition entre pôle de grande production et pôle de production restreinte concerne aussi bien la littérature que les essais (non-fiction). Selon les données de l'Index Translationum, les essais constituent la deuxième catégorie la plus importante de livres traduits, après la littérature (environ la moitié). Ils représentent environ $22 \%$ de l'ensemble des livres traduits (à partir de toutes les langues confondues) entre 1980 et 2002, si l'on additionne les catégories "Droit, éducation, sciences sociales ", "Philosophie, psychologie " et " Histoire, géographie, biographies ", ces catégories mêlant ouvrages scientifiques et ouvrages pour le grand public.

Les variations entre les langues accusent cependant des écarts importants. Elles tiennent en partie à la centralité de la langue : plus une langue est centrale, plus on traduit de catégories ou genres de livres différents de cette langue. Ainsi, la diversité des échanges est moindre en sciences humaines et sociales qu'en littérature, car nombre de langues périphériques n'ont quasiment pas d'ouvrages de sciences humaines traduits dans d'autres langues. Cela tient en partie au fait que les chercheurs les plus internationalisés de ces pays publient directement en anglais.

Pourtant, l'analyse quantitative des flux de traduction en français depuis les années 1980 par langue et catégorie de livres montre que la diversité culturelle, mesurée à travers la langue d'origine, est plus élevée parmi les ouvrages de sciences humaines et de littérature haut de gamme qu'au pôle de grande production, où les best-sellers et les genres situés au pôle de grande diffusion, comme le roman rose ou les thrillers, sont très majoritairement traduits de l'anglais ; il en va de même pour les livres de voyage, les essais d'actualité ou les biographies destinées au grand public. Ainsi, dans les collections de littératures étrangères les plus prestigieuses des grandes maisons d'édition, le nombre de langues traduites peut aller jusqu'à 
une trentaine (pour 40 pays), tandis que l'anglais est sous-représenté par rapport à sa part dans l'ensemble des traductions en français (un tiers contre deux tiers des nouveautés traduites en français entre 1985 et 2002)(8).

Bien que les langues d'origine soient moins diversifiées en sciences humaines et sociales qu'en littérature, on constate la même sous représentation de l'anglais : les traductions faites à partir de l'anglais constituent ainsi un peu plus de la moitié des nouveautés de sciences humaines et sociales traduites en français de 1985 à 2002 à partir de onze langues, contre un quart pour l'allemand, un livre sur dix étant traduit de l'italien et moins d'un sur vingt de l'espagnol, cette part variant en outre fortement selon les disciplines(9). Cependant, dans le cas des sciences humaines et sociales, la question de la diversité linguistique se pose en des termes différents de celui de la littérature, comme on va le voir à présent.

\section{Spécificités des sciences humaines et sociales}

Le mot d'ordre de la " globalisation " tout comme la construction européenne, qui s'est accompagnée de programmes de financement de la recherche au niveau régional, a eu des répercussions dans le domaine des savoirs et de leur circulation à différents niveaux, à en juger par l'intensification des échanges scientifiques internationaux et par l'intense réflexion sur les moyens de " dénationaliser " les sciences humaines et sociales. Toutefois, la suppression des frontières nationales de la pensée n'entraîne pas automatiquement un dialogue pacifique entre les traditions culturelles et leur hybridation comme le voudraient les partisans de la " globalisation " culturelle. Dans cet espace reconfiguré, des rapports de force subsistent, d'ordre politique, économique et symbolique, qui y revêtent des significations spécifiques. Par exemple, si l'on prend comme indicateur d'internationalisation les co-signatures d'articles par des chercheurs de différents pays, on observe une hausse significative de collaborations scientifiques intra-européennes depuis 1990, mais le taux d'augmentation des co-signatures de chercheurs européens avec des chercheurs américains est nettement plus élevé, ce qui reflète I'hégémonie étatsunienne dans le domaine des sciences humaines et sociales(10).

Pour les sciences humaines et sociales, deux questions se posent : celle de la langue d'écriture et celle du support (article ou livre, support papier ou en ligne). La pression sur les chercheurs français est forte de publier en anglais. Mais faut-il écrire directement en anglais ou

(8) Gisèle Sapiro (dir.), Le Marché de la traduction en France à l'heure de la mondialisation, Paris, CNRS Editions, 2008, p. 175-210.

(9) Gisèle Sapiro et loana Popa, "Traduire les sciences humaines et sociales : logiques éditoriales et enjeux scientifiques ", in Gisèle Sapiro (dir.), Le Marché de la traduction en France à I'heure de la mondialisation, ibid.

(10) Yves Gingras et Johan Heilbron, "L'internationalisation de la recherche en sciences sociales et humaines en Europe (1980-2006) ", in Gisèle Sapiro (dir.), L'Espace intellectuel en Europe, op. cit., p. 359-390. 
passer par la traduction ? II y a bien évidemment des arguments en faveur du passage à l'anglais : le recours à une même langue de travail facilite la communication, amoindrit les problèmes de compréhension (et d'erreurs fréquentes dans les traductions), réduit les coûts de traduction. Cependant, il y a aussi de solides arguments contre. En premier lieu, il faut tenir compte de l'inégalité devant la langue anglaise. Deuxièmement, il ne suffit pas d'écrire en anglais pour accéder aux revues anglaises ou américaines : le format des articles, l'argumentation elle-même dépendent de traditions qui varient fortement entre disciplines et entre pays. La troisième objection est d'ordre épistémologique : en sciences humaines et sociales, la traduction constitue un enrichissement parce qu'elle favorise la réflexivité et conduit à dénaturaliser les catégories de la pensée, parfois issues du sens commun - elle constitue ainsi un garde-fou contre la standardisation de la pensée. D'autant que l'écriture des sciences humaines et sociales est moins formalisée que les sciences de la nature, elle implique une dimension historique, fait place à la narration, recourt parfois à un langage littéraire, métaphorique, à des jeux de mots qui peuvent la rendre, sinon intraduisible, en tout cas difficile à transposer dans une autre langue.

La question qui se pose dès lors est celle du financement de ces traductions. En France, le Centre national du livre joue un rôle majeur tant pour l'extraduction d'ouvrages du français que pour leur intraduction, mais son action était réservée jusqu'à une date récente aux livres publiés en tant que tels en français : par exemple, un recueil d'articles publié en traduction sans qu'il existe d'ouvrage en français ne sera pas financé. Or les recueils d'articles ont souvent constitué une manière d'introduire les différentes facettes des travaux d'un auteur à l'étranger : ce fut le cas pour l'œuvre de Bourdieu en Allemagne ou au Brésil(11). Par ailleurs, pour accorder une subvention, le CNL exige - à juste titre - qu'un contrat de traduction ait été signé prévoyant une rémunération décente pour le traducteur, mais, du coup, nombre d'ouvrages de sciences humaines traduits bénévolement dans les pays d'Amérique latine, par exemple, ne peuvent en bénéficier.

Le financement des traductions d'articles en sciences humaines et sociales a été pendant longtemps (et est toujours très largement) à la charge des laboratoires de recherche, dont les crédits sont restreints. Depuis peu, quelques (rares) établissements, comme l'Institut d'études politiques de Paris, ont adopté une politique de prise en charge de la traduction des ouvrages produits par leurs chercheurs, parfois en accord avec une presse universitaire anglaise ou américaine. Récemment, le CNL a mis en place, en collaboration avec le CNRS et avec CAIRN, un plan de financement des traductions en anglais pour les revues de sciences humaines et sociales. Ce plan laisse cependant deux problèmes irrésolus : premièrement, les principes de sélection des revues bénéficiaires du plan éliminent des pans entiers de la recherche

(11) Gisèle Sapiro et Mauricio Bustamante, "Translation as a measure of international consecration. Mapping the world distribution of Bourdieu's books in translation", Sociologica, n² 2-3, 2009. 
française ; deuxièmement, il ne suffit pas d'être traduit pour être lu et cité : tant que les revues françaises n'entreront pas dans le champ de référence des chercheurs étrangers, en particulier américains, ces efforts risquent de se solder par un résultat assez maigre(12). Or si les revues de sciences humaines et sociales françaises sont très peu citées aux Etats-Unis ou au Royaume Uni, comme l'a révélé une enquête menée par le CNRS en 2004, il n'en va pas de même des ouvrages de penseurs ou de chercheurs français, qui sont au contraire très cités en traduction (c'est le cas des Barthes, Foucault, Bourdieu, Derrida, Kristeva et d'autres) ou lorsqu'ils sont directement publiés en anglais (comme dans le cas de Bruno Latour). Ce qui nous conduit à la question du support, puis à celle des disciplines.

Concernant le support, si l'article est la forme la plus adéquate pour l'échange entre pairs dans un domaine de recherche circonscrit et pour le contrôle collectif des savoirs produits, son impact par-delà le milieu des spécialistes est limité. En mettant à part les revues intellectuelles qui ont moins une visée scientifique qu'un objectif de débat de questions de société plus ou moins nourri de la recherche, à l'instar des Temps modernes, d'Esprit ou du Débat, seules quelques grandes revues scientifiques comme Actes de la recherche en sciences sociales ou Les Annales parviennent à toucher un public plus large.

Cette limitation du public des revues est aggravée par la monopolisation du savoir par les grands groupes d'édition scientifique comme Elsevier, Routledge ou autre, qui contrôlent l'accès à ce savoir et en font une source de rentabilité économique. Ces groupes qui possèdent les principales revues en sciences de la nature, dont ils vendent l'abonnement à prix d'or - ce qui a entraîné la réduction drastique des crédits que les bibliothèques anglo-américaines consacraient aux livres -, ont également acquis bon nombre de revues de sciences humaines et sociales en langue anglaise. Le ou la chercheur-e qui publie dans une revue appartenant à l'un de ces groupes est invité à choisir entre deux modalités : l'open access, pour lequel la revue exige une contribution des chercheurs (ou de leurs laboratoires) d'environ 2 000-3 000 euros, ou bien l'accès payant aux lecteurs, qui ne coûtera au chercheur "que " quelques centaines d'euros s'il souhaite acquérir des tirés à part de son article. Ces tarifs exorbitants rendent impossible la diffusion en libre accès pour les sciences humaines et sociales, qui bénéficient de moyens très inférieurs à ceux des sciences de la nature, à moins d'opter pour la mise en ligne de l'article en version non formatée comme le font la plupart des chercheurs qui protestent contre cette monopolisation du savoir (voir à ce propos la charte adoptée par les professeurs de Harvard). En France, le modèle proposé par le portail Cairn, qui consiste à rendre l'accès aux articles libre au bout de trois ans d'exploitation à des prix raisonnables, a permis de trouver un équilibre entre la viabilité économique des revues et la nécessité de restituer les résultats

(12) Yves Gingras et Sébastien Mosbah-Natanson, " La question de la traduction en sciences sociales : les revues françaises entre visibilité internationale et ancrage national ", Archives européennes de sociologie, vol. 51, $n^{\circ} 2,2010$, p. 305-321. 
de la recherche à la société qui la finance et d'en garantir l'impact le plus large possible. À quoi s'ajoute la très précieuse entreprise patrimoniale de mise en ligne des anciens numéros des revues de sciences humaines et sociales par le portail Persée.

Ces limites structurelles ou conjoncturelles que rencontrent les revues scientifiques du point de vue de leur diffusion font qu'à rebours de la tendance à relativiser la place du livre dans l'évaluation de la production des chercheurs suivant le modèle des sciences de la nature, il est plus que jamais nécessaire de réaffirmer le rôle majeur qu'il joue dans la restitution et la diffusion des savoirs en sciences humaines et sociales, en particulier hors du monde académique, et dans la circulation internationale des idées. L'« impact " des sciences humaines et sociales sur la société en dépend. A fortiori en Europe, où il existe une tradition de publication des ouvrages de sciences humaines et sociales dans l'édition généraliste, à la différence des États-Unis et du Royaume Uni, où ils sont confinés au secteur académique.

Certes, le rôle du livre est variable selon les disciplines : il est central dans les disciplines les plus " littéraires ", comme la philosophie, les études littéraires, l'histoire, et décroît à mesure qu'on va vers les disciplines qui ont adopté le modèle des sciences de la nature comme la psychologie ou l'économie. La sociologie et l'anthropologie occupent une position intermédiaire entre ces deux pôles.

L'édition est un des lieux de médiation entre contraintes économiques, politiques et culturelles. En effet, si la circulation internationale des ouvrages de sciences humaines et sociales est relativement autonome des logiques de marché et des logiques idéologiques, elle n'y échappe pas complètement.

Du point de vue idéologique, la circulation des livres de sciences humaines peut s'inscrire dans des perspectives militantes - que l'on pense au rôle du parti communiste dans les traductions des textes de Marx et du marxisme ou, plus près de nous, à celui des petits éditeurs critiques(13). Elle peut être également soutenue par des politiques étatiques en vue de favoriser le rayonnement de la culture nationale à l'étranger ou la diplomatie d'influence. Par exemple, le gouvernement américain a aidé les traductions des ouvrages de la pensée libérale (comme Hayek) à destination des pays d'Europe de l'Est pendant la période communiste, puis vers les pays arabes. Il a également mis en œuvre une politique de rachat des traductions de l'anglais en français pour les diffuser à bas prix dans les zones francophones en Afrique. Les programmes européens ont également soutenu la production et la circulation de travaux sur l'Europe.

Du point de vue éditorial, les sciences humaines et sociales relèvent, on l'a vu, de la catégorie des essais. Mais au sein de cette catégorie, elles se situent dans le circuit de rotation lente, à

(13) Sophie Noël, "L'engagement par la traduction. Le rôle des petits éditeurs indépendants dans l'importation des ouvrages de sciences humaines ", in Gisèle Sapiro (dir.), Traduire la littérature et les sciences humaines. Conditions et obstacles, Paris, DEPS/ministère de la Culture, 2012, p. 273-293. 
la différence de nombre d'essais destinés au grand public liés à l'actualité ou des biographies. Du fait de leur faible rentabilité, elles subissent un processus de sélection sévère sur le marché de la traduction. D'autant que la pression économique s'est accrue également dans le secteur académique : les presses universitaires anglo-américaines sont elles-mêmes de plus en plus soumises à des impératifs de rentabilité(14).

La sélection des livres s'opère selon deux principaux critères : le nom de l'auteur, à savoir son capital symbolique (tous les ouvrages des auteurs de la French Theory sont automatiquement traduits) ; l'intérêt du thème (par exemple, les éditeurs américains s'intéressent particulièrement à tout ce qui touche à l'islam ; pendant un certain temps, la gastronomie a été un thème à la mode). Ces critères ne répondent pas à des logiques purement économiques, mais ils relèvent en partie de logiques culturelles ou intellectuelles. Un auteur fortement doté en capital symbolique peut continuer à être traduit même si les espoirs de ventes ne sont pas très élevés, dans une optique d'accumulation de capital symbolique et de patrimonialisation. D'autres facteurs implicites qui interviennent dans le processus de sélection relèvent également de logiques culturelles, tels que le capital symbolique de la langue et de la tradition nationale dans le domaine concerné, ou le capital symbolique de l'éditeur (par exemple, alors qu'en littérature, Gallimard jouit d'un prestige inégalé auprès des éditeurs étrangers, comme en témoigne le fait que la maison concentre $29 \%$ des traductions du français réalisées aux États-Unis entre 1990 et 2003, la part de toutes les autres étant inférieure à $8 \%$, dans le domaine des sciences humaines et sociales, Le Seuil bénéficie d'un crédit supérieur)(15).

Du point de vue culturel, la circulation internationale des livres de sciences humaines et sociales dépend à la fois du champ intellectuel et du monde universitaire. L'espace intellectuel transnational n'est pas moins régi par des rapports de force, mais selon des logiques qui lui sont propres. C'est en premier lieu le capital symbolique accumulé par certaines traditions nationales qui joue, de manière variable selon les disciplines : par exemple, la philosophie allemande, la pensée théorique française ou l'anthropologie anglo-américaine jouissent d'un prestige supérieur à celui des autres traditions dans ces domaines. Ce capital symbolique se reflète dans les variations des flux de traduction entre les disciplines. Ainsi, sur les quelque 3000 nouveautés de sciences humaines et sociales traduites en français entre 1985 et 2002, l'anglais représente la moitié, l'allemand $25 \%$, l'italien $10 \%$; cependant, pour les livres de philosophie, les traductions de l'allemand arrivent en tête (près de la moitié), alors que l'anglais est sous-représenté(16). En effet, pendant longtemps, en France, la philosophie américaine a souffert d'une

(14) John Thompson, Books in the Digital Age. The Transformation of Academic and Higher Education Publishing in Britain and the United States, Cambridge/Malden, Polity Press, 2005.

(15) Gisèle Sapiro, "Revaloriser la traduction dans un environnement hostile : le marché éditorial aux États-Unis ", in Gisèle Sapiro, Traduire la littérature et les sciences humaines, op. cit., p. 57-101.

(16) Gisèle Sapiro et loana Popa, "Traduire les sciences humaines et sociales : logiques éditoriales et enjeux scientifiques ", in Gisèle Sapiro (dir.), Le Marché de la traduction en France à l'heure de la mondialisation, op. cit. 
absence de reconnaissance qui entravait les éventuels projets de traduction(17). À l'inverse, la diffusion mondiale des penseurs français labellisés sous l'étiquette de la French Theorytient en bonne partie à leur réception aux États-Unis et à l'usage critique qui en a été fait dans le champ académique étatsunien(18), ce qui témoigne de la position dominante des États-Unis dans l'espace intellectuel transnational et de son rôle médiateur sur le marché de la traduction.

La circulation des savoirs n'est cependant pas un phénomène mécanique. Comme le rappelle Pierre Bourdieu, reprenant une phrase de Marx, les textes circulent sans leur contexte, ce qui génère des malentendus(19). Par-delà les différences de découpage disciplinaires, qui font qu'un Habermas, sociologue en Allemagne, est reçu en France comme philosophe, les textes traduits font l'objet de réappropriations, de marquages (collection, préface, postfaces), d'annexions, dans un processus qui doit beaucoup aux logiques propres à l'espace de réception. Les auteurs traduits peuvent être instrumentalisés dans des luttes internes au champ intellectuel considéré, comme l'illustre l'exemple de la réception de l'œuvre de John Rawls en France(20). Les importateurs appartenant au champ académique jouent dans le cas des sciences humaines et sociales un rôle encore plus important que pour la littérature, à toutes les étapes de la circulation, depuis l'intervention auprès des éditeurs dans le processus de sélection (pour proposer, donner des avis) jusqu'à l'enseignement, en passant par la traduction elle-même, la recension, la citation et l'usage pour la recherche.

Malgré l'accélération des échanges, la circulation des ouvrages de sciences humaines et sociales conserve une temporalité qui lui est propre et qui induit des effets de décalage dans les processus de réception, dont il faut tenir compte lorsqu'on étudie la circulation des savoirs(21). On se contentera d'un exemple, celui de la réflexion sur le colonialisme et l'impérialisme, dont la circulation est fortement liée aux conjonctures politiques. Elle est apparue dans le cadre des luttes contre le colonialisme à la fin du xixe et s'est développée dans les années 1950-60 en France et en Allemagne, au moment de la décolonisation. Or les auteurs de cette période, comme Frantz Fanon, qui ont été marginalisés dans le champ intellectuel français à partir des années 1980, sont devenus des références aux États-Unis dans les années 1990 pour penser le post-colo-

(17) Romain Pudal, « La philosophie américaine existe-t-elle ? Les obstacles à l'importation du pragmatisme en France n in Gisèle Sapiro (dir.), Traduire la littérature et les sciences humaines, op. cit., p. 321-342.

(18) François Cusset, French Theory. Foucault, Derrida, Deleuze \& Cie et les mutations de la vie intellectuelle aux États-Unis, Paris, La Découverte, 2003.

(19) Pierre Bourdieu, "Les conditions sociales de la circulation internationale des idées ", Actes de la recherche en sciences sociales, $n^{\circ} 145$, p. 3-8.

(20) Mathieu Hauchecorne, "Une réception politisée. La traduction de John Rawls et de la philosophie politique et morale anglophone en français ", in Gisèle Sapiro (dir.), Traduire la littérature et les sciences humaines, op. cit., p. 343-368.

(21) Pour un cas extrême, voir Marc Joly, Devenir Norbert Elias, Paris, Fayard, 2012 et Id. "La "grande œuvre" méconnue : Norbert Elias en France ", in Gisèle Sapiro (dir.), Traduire la littérature et les sciences humaines, op. cit., p. 299-320. 
nialisme, en lien étroit avec le regard critique porté sur la politique étatsunienne dans le monde. La publication en 2000 du livre de Hardt et Negri intitulé Empire a suscité une vaste réflexion sur l'impérialisme aux États-Unis. Et la réflexion sur le post-colonialisme et l'impérialisme est revenue par ce biais en France depuis plusieurs années...

Pour conclure, la réflexion sur la circulation des ouvrages de sciences humaines et sociales doit prendre en compte ses spécificités, aussi bien au niveau des problèmes qui se posent à la traduction dans ce domaine qu'au niveau des conditions sociales de sa circulation. On a évoqué, parmi ces dernières, la nécessité de réaffirmer l'importance du livre, qui joue un rôle majeur dans les échanges scientifiques et intellectuels internationaux, tout en assurant la diffusion du savoir auprès d'un public transcendant le cercle des pairs et des spécialistes. Mais l'inscription des ouvrages de sciences humaines et sociales dans le champ éditorial et dans l'édition généraliste en particulier la soumet aux contraintes commerciales qui pèsent sur ces espaces. D'où la nécessité du recours à des subventions pour garantir la viabilité économique des projets de traduction dans ce domaine. La traduction en sciences humaines et sociales soulève d'autres problèmes spécifiques qu'il n'a pas été possible de développer dans le cadre de cet article, en particulier celui de la formation et de la spécialisation des traducteurs, qui est nécessaire à une transposition adéquate et qui peut être de ce fait même incompatible avec la professionnalisation. De façon plus générale, on pourrait préconiser la formation à la traduction dans toutes les disciplines de sciences humaines, non pas forcément pour en faire un métier, mais comme une pratique intellectuelle parmi d'autres, comme le commentaire ou le compte rendu critique, qui est indispensable non seulement à la circulation des idées, mais aussi à une démarche réflexive favorisant le questionnement et la relativisation de nos catégories de pensée.

\section{sapiro@msh-paris.fr}

Gisèle Sapiro est directrice de recherche au CNRS et directrice d'études à l'EHESS. Elle dirige le Centre européen de sociologie et de science politique (Univ. Paris 1-EHESS-CNRS). Spécialiste de sociologie des intellectuels, de la littérature, de la traduction, des échanges culturels internationaux, ainsi que d'épistémologie des sciences sociales, elle est l'auteure de La Guerre des écrivains, 1940-1953 (Fayard, 1999 ; à paraître en anglais chez Duke UP) et de La Responsabilité de l'écrivain. Littérature, droit et morale en France xIXe-xx|e siècle (Seuil 2011 ; à paraitre en espagnol chez Eudeba-UNL), ainsi que de nombreux articles, parus dans une douzaine de langues. Elle a également dirigé ou codirigé plusieurs ouvrages : Pour une histoire des sciences sociales (Fayard, 2004), Pierre Bourdieu, sociologue (Fayard, 2004 ; traduit en espagnol chez Nueva Vision) ; Translatio. Le marché de la traduction en France à l'heure de la mondialisation (CNRS Editions, 2008) ; Les contradictions de la globalisation éditoriale (Nouveau Monde, 2009) ; L'Espace intellectuel en Europe (La Découverte, 2009) ; Traduire la littérature et les sciences humaines : conditions et obstacles (DEPS ministère de la Culture). 